\title{
Vortical Gusts: Experimental Generation and Interaction with Wing
}

\author{
Esteban A. L. Hufstedler* \\ Catholic University of Louvain, 1348 Louvain-la-Neuve, Belgium \\ and \\ Beverley J. McKeon \\ California Institute of Technology, Pasadena, California 91125 \\ DOI: $10.2514 / 1 . J 056914$
}

\begin{abstract}
We describe the experimental generation of isolated vortical gusts and the interaction between these gusts and a downstream airfoil at a Reynolds number of 20,000. A standard method of generating a vortical gust has been to rapidly pitch an airfoil. A different approach is presented here: heaving a plate across a tunnel and changing direction rapidly to release a vortex. This method is motivated by the desire to limit a test article's exposure to the wake of the gust generator by moving it to the side of the tunnel. Two suites of experiments were performed to characterize the performance of the gust generators and to measure the forces on and flow around the downstream airfoil. The novel mechanism allowed for measurement of the resumption of vortex shedding from the downstream airfoil, which was impossible with the pitching generator.
\end{abstract}

\section{Nomenclature}

$C_{L}$

$c_{a}$

$c_{p}$

$h$

$R e_{c}$

$S$

$T$

$t$

$t_{a}$

$t_{\text {accel }}$

$t_{c}$

$t_{\mathrm{cp}}$

$t_{\text {heave }}$

$t_{p}$

$t_{\text {pitch }}$

$U$

$u$

$V_{\text {heave }}$

$v$

$x$

$x^{\prime}$

$y$

$y_{0}$

$y_{\text {peak }}$

$y_{\text {plate }}$

$=$ time, $\mathrm{s}$

$=$ plate acceleration time, $\mathrm{s}$

$=$ heaving time, $\mathrm{s}$

$=$ freestream speed, $\mathrm{m} / \mathrm{s}$

$=$ plate heaving speed, $\mathrm{m} / \mathrm{s}$ process, $\mathrm{m}$ midline, $\mathrm{m}$ heaving plate, $\mathrm{m}$ speed ratio, $V_{\text {heave }} / U$

$=$ heaving time ratio, $t_{\text {heave }} / t_{\mathrm{cp}}$

$=$ normalized plate acceleration time, $t_{\mathrm{accel}} / t_{\mathrm{cp}}$

$=$ chordwise convective time across airfoil, $\mathrm{s}$

$=$ chordwise convective time across plate, $\mathrm{s}$

$=$ normalized airfoil pitching time, $t_{\text {pitch }} / t_{c}$

$=$ generator pitching time, $\mathrm{s}$

$=$ velocity in $x$ direction, $\mathrm{m} / \mathrm{s}$

$=$ velocity in $y$ direction, $\mathrm{m} / \mathrm{s}$

$=$ streamwise coordinate, $\mathrm{m}$

$=$ streamwise position computed from unwrapping

$=$ cross-stream coordinate with respect to tunnel

$=$ initial plate distance from midline, $\mathrm{m}$

$=$ cross-stream position of maximum displacement of

$=$ cross-stream position of heaving plate, $\mathrm{m}$

Presented as Paper 2016-4257 at the AIAA 46th AIAA Fluid Dynamics Conference, Washington, D.C., 13-17 June 2016; received 15 November 2017; revision received 18 August 2018; accepted for publication 18 December 2018; published online 29 January 2019. Copyright (C) 2019 by Esteban A. L. Hufstedler and Beverley J. McKeon. Published by the American Institute of Aeronautics and Astronautics, Inc., with permission. All requests for copying and permission to reprint should be submitted to CCC at www.copyright.com; employ the ISSN 0001-1452 (print) or 1533-385X (online) to initiate your request. See also AIAA Rights and Permissions www.aiaa.org/randp

*Graduate Student, Thermodynamics and Fluid Mechanics Department; also Post-Doctoral Researcher, California Institute of Technology, Pasadena, California 91125; Esteban.Hufstedler@ uclouvain.be.

†Theodore von Kármán Professor of Aeronautics, Graduate Aerospace Laboratories of the California Institute of Technology; McKeon@ caltech.edu. Associate Fellow AIAA.

\begin{tabular}{|c|c|c|}
\hline$y_{\text {upstre }}$ & $=$ & \\
\hline$z$ & & \\
\hline$\alpha$ & $=$ & of attack of test article, rad (unless otherwise \\
\hline$\alpha_{1}$ & $=$ & $\begin{array}{l}\text { angle of pitching gust generator, rad (unless } \\
\text { ise noted) }\end{array}$ \\
\hline$\alpha_{2}$ & $=$ & itching gust generator, rad (unless \\
\hline$\alpha_{\text {eff }}$ & $=$ & $\begin{array}{l}f \text { attack of heaving gust generator, } \\
\text { wise noted) }\end{array}$ \\
\hline$\alpha_{\text {upstr }}$ & $=$ & $\begin{array}{l}\mathrm{k} \text { of pitching gust generator, rad } \\
\text { se noted) }\end{array}$ \\
\hline$\Gamma_{v}$ & - & $\mathrm{x}, \mathrm{m}^{2} / \mathrm{s}$ \\
\hline$\Gamma_{v \text {,est, heave }}$ & $=$ & $\begin{array}{l}\text { estimated circulation of shed vortex from heaving } \\
\text { generator, } \mathrm{m}^{2} / \mathrm{s}\end{array}$ \\
\hline$\Gamma_{v, \text { est,pitch }}$ & $=$ & $\begin{array}{l}\text { estimated circulation of shed vortex from pitching } \\
\text { generator, } \mathrm{m}^{2} / \mathrm{s}\end{array}$ \\
\hline$\Gamma_{2}$ & - & vo \\
\hline$\Delta x$ & $=$ & $\begin{array}{l}\text { streamwise distance between generator and test } \\
\text { article, } m\end{array}$ \\
\hline & & normalized time, $t / t_{c}$ \\
\hline$\omega_{z}$ & $=$ & vorticity measured in $x-y$ plane, $\mathrm{s}$ \\
\hline
\end{tabular}

\section{Introduction}

IRCRAFT and creatures with wings agree: gusts are important. A Were the world simpler, creatures and craft could fly through a world without variations in the wind's velocity and without the complicated structural and aerodynamic effects that such variations incur. Strong, unexpected gusts can lead to the failure of overloaded structures or a loss of flight control. Within the broader field of unsteady aerodynamics, a body of research has focused on the aerodynamic response of wings to natural and man-made gusts. These include the unsteady flows around large buildings [1], in the airwake of ships [2], or incidental interactions with the wakes of aircraft $[3,4]$ or rotors [5], including wind turbines. There has also been considerable effort invested into the creation of analogs of those gusts for further research, as outlined in the following. Of related interest has been the burgeoning field of controlled analogs of those gusts or simple transients to provide understanding for more complex realistic flows. Examples are the study of airfoil response to maneuvers such as pitching, plunging, and/or surging, as reviewed in, e.g., [6]. In each of these cases, goals include a broader understanding of the time scales and force magnitudes associated with unsteady flow development and, of primary importance to this study, improved aerodynamic performance under periodic vortex passage and random 
gust encounters. Though this Paper focuses on the generation of, and interaction with, vortical gusts, this Introduction will briefly survey classical and recent research on related unsteady flows as well.

The early review of natural unsteadiness in the atmosphere by Donely [7] showed that atmospheric gusts are roughly independent of direction. The velocity profiles of these gusts were well modeled as sinusoidal or triangular functions. Etele [8] classified the following scales of unsteady wind as part of a modeling effort: geostrophic, atmospheric boundary layer, and small-scale random turbulence. Two commonly used models of continuous gusts are the Dryden [9] and von Kármán [10] wind turbulence models, which used the power spectral densities of measured atmospheric turbulence to specify stochastically variable velocities in three dimensions. In contrast to these models of continuous gusting, many studies of unsteady aerodynamics use simplified models of gusts, such as the Federal Aviation Administration's [11] definition of a discrete gust as a change in the flow's velocity scaled with one period of a one-minuscosine function. Knigge and Raasch [12] extracted gusts from largeeddy simulations, yielding one-dimensional gusts that are wider than the one-minus-cosine standard. Other simple, traditionally used gusts include impulsive, ramped, or harmonic changes in the freestream or transverse velocities. These examples, among others, are described in this Introduction, but this Paper does not attempt an exhaustive list of the freestream perturbations that have been studied.

The theoretical underpinnings of unsteady aerodynamics began as extensions of steady two-dimensional thin airfoil theory [13]. These works had the same limitations as their foundation: thin airfoils; attached, inviscid, incompressible flow on the body; small angles of attack; a zero-thickness wake that leaves the body at a sharp trailing edge and proceeds downstream with no vertical deflection; and irrotational flow outside of the body and wake. Such approaches incorporate knowledge of the physical flow in two ways: viscosity is the physical cause of flow smoothly leaving the sharp trailing edge of an airfoil as well as the cause of its wake. A number of eponymic formulas were found to describe the response of an airfoil to gusts. The Wagner [14] function describes its change in lift over time due to an impulsive change in the angle of attack. The Theodorsen [15] function describes the forces on a wing undergoing harmonic oscillation. Küssner [16] found the response to an impulsive transverse gust. Sears and von Kármán [17] analyzed the response of an airfoil to a harmonic vertical gust. Extensions of thin airfoil theory continue to the present, including a conservation of impulse approach to semi-analytically model the evolution of an airfoil's wake, discretized into point vortices, by Tchieu and Leonard [18].

A number of classical gusts, and approximations to them, have been studied experimentally. The transverse sharp-edged gust problem has been examined for decades, and there has been a renewed interest in this problem in recent years. One of the earliest means was a NACA gust tunnel, which launched model aircraft with a catapult over a vertical blower $[\underline{7,19]}$ to simulate transverse gusts. These early studies found reasonable matches between theory and experiments. Analogously, Perrotta and Jones [20] used a set of jets mounted on the bottom of a tow tank to provide a limited region of transverse velocity. These experiments found that classical analytical models were insufficient for modeling the forces in strong gust encounters that involve separated flows and large angles of attack, especially during the flow's recovery from the gust interaction. Recent work by Biler et al. [21] in the same facility found good agreement between experimental and numerical results at a Reynolds number based on the chord length and freestream velocity of $R e_{c}=40,000$ and confirmed the complexity of such gust encounters. Another classical unsteady problem, the accelerated flat plate, has been investigated by a number of researchers. A collaborative paper by Stevens et al. [22] showed strong agreement between experiments and simulations, including the evolution of the leading- and trailing-edge vortices, and small effects of varying $R e_{c}$ over the range of 600 to 20,000 .

The problem of a harmonic gust has also been examined experimentally and numerically. The typical method $[23,24]$ of creating such gusts is to pitch vanes upstream of the test section to yield transverse gusts or to open and close shutters downstream of the test section [25] to create an oscillating freestream. The use of a small number of vanes $[26,27]$ can create distinct vortices, which yield a transverse velocity far from the vortices. Golubev et al. [28] numerically modeled two-dimensional sharp-edged and harmonic variations in the oncoming flow interacting with an airfoil at $R e_{c}=10,000$. For low wing loading, the responses to oscillating streamwise and transverse flows matched inviscid predictions, apart from some low-frequency oscillations that appeared to create a large dynamic-stall effect. Baik et al. [29,30] found a good match with theory for pitching and plunging airfoils, as an analog for oscillating flows, except when leading-edge separation was present. The circulation shed by flapping wings has also been an active topic of research. Buchholz et al. [31] used a kinematic scaling to relate the pressure gradients on the wing to the circulation released over one period of motion.

Much of the research on isolated vortex-wing interactions has been performed numerically. Golubev et al. $[\underline{28}, \underline{32}, \underline{33}]$ simulated vortex interactions at $R e_{c}=10,000$ and 60,000 , in which the size of the vortex was comparable to that of the airfoil. These simulations found that larger, stronger, closer vortices resulted in larger resulting forces on the airfoil, even up to creating stall-like conditions. The inclusion of three-dimensionality in some simulations reduced the oscillations in the forces but did not change the general behavior. The effect of a finite aspect ratio on the vortex-wing interaction was numerically studied by Gordnier and Visbal [34], particularly focusing on head-on collisions in transitional flow at $R e_{c}=200,000$. The collision split the vortex, disturbing both the upper and lower boundary layers. The typical flow around the airfoil reasserted itself over roughly eight convective time units. Barnes and Visbal [35] computationally examined a spatially periodic interaction with a wing-parallel vortex in a flow similar to that of the previous study and found complex behavior due to the laminarto-turbulent transition. This included the generation of leading-edge vortices from laminar separation bubbles, resulting in a vortex dipole forming with the gust. The response of a wing to large vortices at $R e_{c}=10,000$ and 60,000 was simulated by Nguyen et al. [36], who found that inviscid models more accurately predicted the forces when the gusts were of sufficiently high amplitude. With weaker gusts, viscous effects were more prominent. Barnes and Visbal $[37,38]$ simulated isolated vortex-wing interactions at $R e_{c}=150,000$ and 200,000. These studies showed important effects of the airfoil's angle of attack: the lift returned to its preinteraction state in approximately 2 convective time units when at zero incidence and approximately 10 time units at $12 \mathrm{deg}$.

This Paper proposes and investigates a new kind of vortex generator: a flat plate that moves transversely across the test section and changes direction to release a vortex. By releasing a vortex and moving away, this novel vortex generator moves its wake to the side of the tunnel. In contrast, the wake of a standard pitching gust generator continues to interact with the test article. This Paper describes the experimental generation of vortical gusts using both types of gust generators, characterizes and compares the subsequent gusts, and details how the gusts interacted with a downstream airfoil. The experiments were performed at a chordwise Reynolds number of 20,000 , which is high enough that simulations across a wide range of parameters are prohibitively expensive, whereas similar experiments can be completed relatively quickly.

The Paper is organized in the following manner. The experimental equipment is introduced with a summary of the test matrix in Sec. II. The properties of the gust generators are characterized and compared in Sec. III. The generators are then used in conjunction with a downstream airfoil to characterize its response to parallel gusts in Sec. IV. Finally, a summary and conclusions are given in Sec. V. Additional details, experiments, and discussion can be found in Hufstedler's thesis [39].

\section{Experimental Approach and Methods}

The essential details of the facility, the pitching and heaving gust generators, the downstream static airfoil model, and the diagnostics and data analysis techniques are given in the following. 

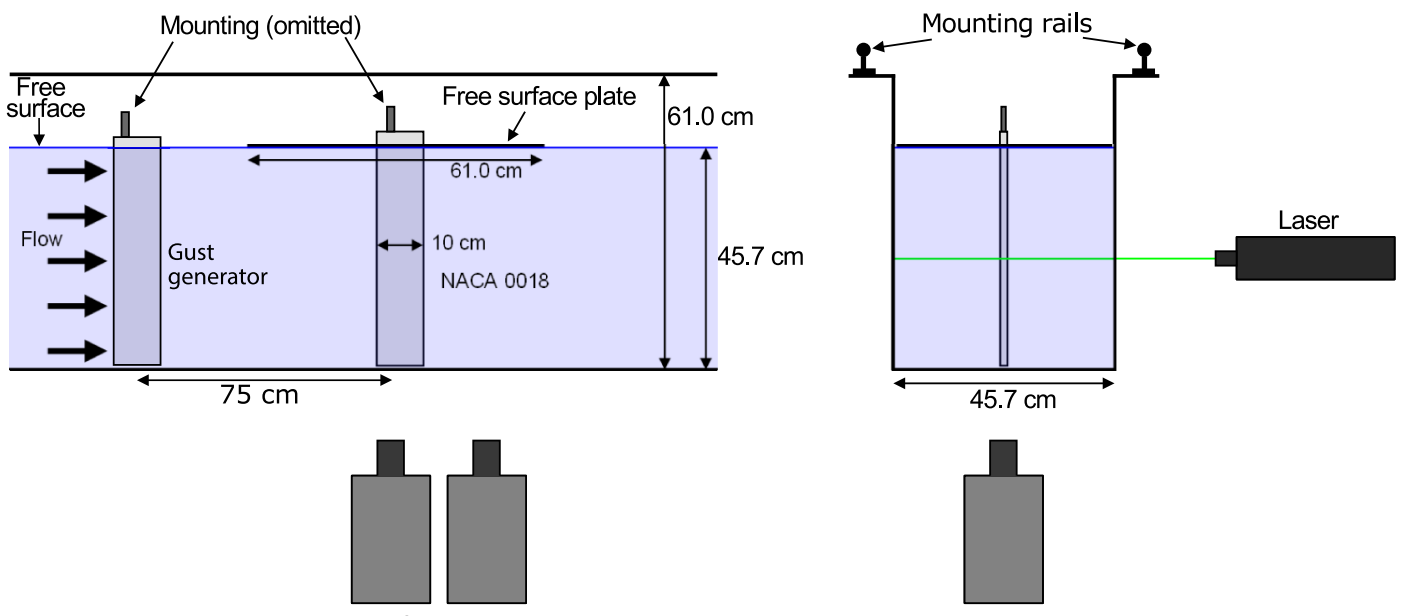

Cameras

Fig. 1 Side view and cross-section of the free surface water tunnel test section and PIV hardware configuration.

\section{A. Facility}

The experiments were performed in a recirculating free surface water tunnel at the California Institute of Technology. A schematic of the tunnel is shown in Fig. 1. The test section is $46 \mathrm{~cm}$ wide and $150 \mathrm{~cm}$ long, with the water $46 \mathrm{~cm}$ deep for this campaign, and its walls and bottom are transparent Plexiglas for visibility. Before entering a 4:1 contraction, the flow passes through two 7.6-cm-thick honeycomb panels and three fine mesh screens to reduce the turbulence in the flow. Rails on the top edges of the test section were used to mount the two gust generators, a static airfoil, and a free surface plate. The tunnel was operated with a pump frequency of $12.5 \mathrm{~Hz}$, yielding a freestream velocity of $U=20 \mathrm{~cm} / \mathrm{s}$. The coordinate system in this Paper uses $x$ as the streamwise direction, $z$ as the vertical spanwise direction, and $y$ as the direction normal to both the freestream and span. The origin is located along the streamwise centerline of the test section.

A computer-controlled gantry system was used to actuate both gust generators. The system was a modification of a kit from CNC Router Parts, using NEMA 34 stepper motors with $6.8 \mathrm{~N} \cdot \mathrm{m}$ of holding torque to actuate the three linear axes and a NEMA 23 motor for the angle of attack.

\section{B. Airfoil Model}

The static test article was a NACA 0018 airfoil with a $10 \mathrm{~cm}$ chord and $48.3 \mathrm{~cm}$ span, wetted to $46 \mathrm{~cm}$. To reduce its reflectivity in particle image velocimetry (PIV) experiments, the wing was lightly sanded, spray painted black, and lightly sanded again. The airfoil passed through, but did not touch, a free-surface plate that extended
2.5 chord lengths up- and downstream of the airfoil and across the width of the tunnel in order to reduce free-surface effects. When appropriate, the airfoil was mounted in the center of the tunnel, $75 \mathrm{~cm}$ downstream of the gust-generation system, in order to measure the effects of the gusts. This distance of 7.5 chord lengths was chosen to reduce the short-range effects of the gust generators, such as local flow deflection by the pitching generator at its final angle of attack. The use of a significantly longer distance was infeasible due to the finite length of the test section. The response of the downstream airfoil to the effects of each gust generator was measured at three angles of attack: $\alpha=0,5$, and $10 \mathrm{deg}$.

\section{Pitching Gust Generator}

The pitching gust generator (PGG) consisted of a NACA 0018 airfoil with a chord length $c_{a}=10 \mathrm{~cm}$ and $55 \mathrm{~cm}$ span, wetted to $46 \mathrm{~cm}$. Its axis of rotation was through its quarter-chord point. A schematic of the PGG's place in the water tunnel is shown in Fig. 2a. The distance to the test article was $\Delta x=75 \mathrm{~cm}$. The field of view of the PIV system is shown as the dashed rectangle. The diagram also displays the parameters required to describe the trajectory of the PGG: its $y$ position $y_{\text {upstream }}$ and its initial and final angles of attack $\alpha_{1}$ and $\alpha_{2}$. The angle of attack trajectory for the PGG is defined as a function of time $t$ in Eqs. (1) and (2),

$$
\alpha_{\text {upstream }}\left(\tau_{a}\right)=\alpha_{1}+\frac{1}{2}\left(\alpha_{2}-\alpha_{1}\right)\left(1+\tanh \left(\frac{k \tau_{a}}{t_{p}}\right)\right)
$$

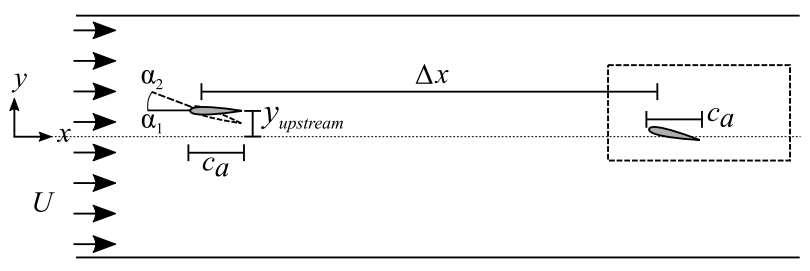

a)

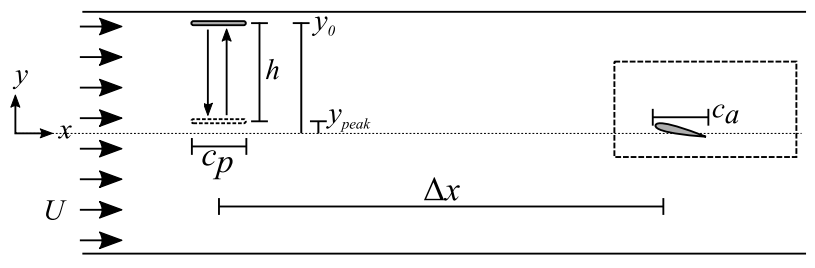

c)

Fig. 2 Diagrams of the a) pitching and c) heaving gust-generation systems and their respective trajectories in b) and d).

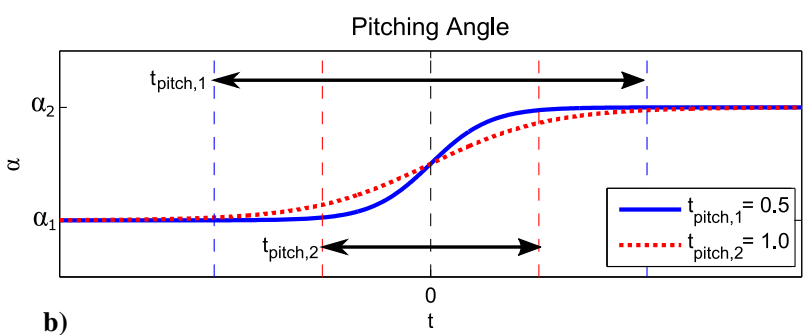

Plate Position

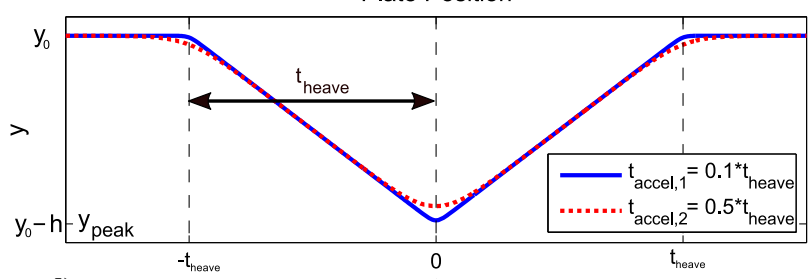

d) 
with

$$
\begin{gathered}
t_{c}=c_{a} / U \\
t_{p}=t_{\mathrm{pitch}} / t_{c} \\
\tau_{a}=t / t_{c} \\
k=2 \operatorname{arctanh}(19 / 20)
\end{gathered}
$$

where time $t_{\text {pitch }}$ is defined as the period for the PGG to complete 95\% of its motion. In all cases, $\alpha_{1}=0$. Two examples of this motion, with different pitching times, are shown in Fig. $2 \mathrm{~b}$.

To characterize the PGG's performance, gusts were created with different pitching amplitudes and examined using a PIV field of view downstream of the PGG, as it pitched from $\alpha_{1}=0$ deg to $\alpha_{2}=-5, \quad-10$, and -13 deg over one convective time unit $t_{c}$. This pitching time was near the minimum achievable with the actuation method. Each experiment was repeated five times. A second set of experiments measured the forces on, and flow around, the downstream airfoil as it interacted with gusts created by the PGG pitching from 0 to -13 deg over $1 t_{c}$, with the PGG at two different upstream positions: $y_{\text {upstream }}=0$ and $0.5 c_{a}$. Additional experiments measured the forces on the test article in response to the PGG at the center of the tunnel, held statically at $-13 \mathrm{deg}$ or $0 \mathrm{deg}$.

\section{Heaving Gust Generator}

The experiments with the heaving gust generator (HGG) employed two flat aluminum plates. These had chord lengths of $c_{p}=5.08$ and $10.16 \mathrm{~cm}$, were $0.64 \mathrm{~cm}$ thick with semicircular leading and trailing edges, and extended in span above the waterline. The resulting thickness ratios of the plates were $1: 8$ and $1: 16$. A schematic of the heaving plate's relative location in the water tunnel and key trajectory parameters is shown in Fig. 2c. In these experiments, the plate started and finished its motion at $y_{0}$.

The trajectory of the HGG was specified by a version of Eldredge's smooth motion equation [40]. This allows for nearly constant speed over most of the motion but is smoothed in the corners to reduce the magnitude of acceleration:

$$
y_{\text {plate }}(t)=y_{0}-\frac{c_{p} t_{a} S}{2 k} \log \left(1+\sinh \left(\frac{k T}{t_{a}}\right)^{2} \cosh \left(\frac{k \tau}{t_{a}}\right)^{-2}\right)
$$

The three key parameters governing the motion are the dimensionless heaving time $T$, the dimensionless heaving speed $S$, and dimensionless acceleration time $t_{a}$, given by

$$
\begin{gathered}
T=t_{\text {heave }} / t_{\mathrm{cp}} \\
S=\left|V_{\text {heave }}\right| / U \\
t_{a}=t_{\text {accel }} / t_{\mathrm{cp}}
\end{gathered}
$$

with

$$
\begin{gathered}
t_{\mathrm{cp}}=c_{p} / U \\
\tau=t / t_{\mathrm{cp}}
\end{gathered}
$$

The parameter $k=2 \operatorname{arctanh}(19 / 20)$ was selected such that $95 \%$ of the velocity change occurs over the acceleration period $t_{\text {accel }}$. The initial and final position of the HGG is $y_{0}$, and the position of the plate where it changes directions is $y_{\text {peak }}$. The trajectory can alternatively be parameterized with the heaving distance, $h=S T c_{p}$. Examples of this trajectory with different values of $t_{a}$ are shown in Fig. 2 d.

Three suites of PIV experiments were performed to characterize the HGG's performance. Experiment HGG-1 explored the parameter space of the heaving plate using both lengths of the plates and varying the heaving distance and speed. Each combination of
$S=[0.09,0.18,0.27]$ and $h=[1.4,1.9,2.4] c_{a}$ was repeated five times, including use of both the 5 and $10 \mathrm{~cm}$ plates, with $y_{0}$ of $1.5 \mathrm{~cm}$ from the $+y$ side of the tunnel. The acceleration time was $0.72 t_{\mathrm{cp}}$ for the $10 \mathrm{~cm}$ plate and $1.44 t_{\mathrm{cp}}$ for the $5 \mathrm{~cm}$ plate.

For comparison with the gusts from the PGG, experiment HGG-2 measured the generated gusts for $S=0.09$ and $0.25, y_{\text {peak }}=0$, and each experiment was repeated five times. Here, $y_{0}$ was $3.8 \mathrm{~cm}$ from the edge of the tunnel. The acceleration time was $t_{\text {accel }}=0.50 t_{\mathrm{cp}}$. Further experiments (HGG-3) investigated the interaction between the $5 \mathrm{~cm}$ HGG's gusts and the test article. It was tested with combinations of both initial directions of motion, $S=[0.09,0.25]$, and the peak position of the plate was $y_{\text {peak }}=[0, \pm 0.5, \pm 1.0] c_{a}$. Experiments with simultaneous PIV and force measurement were repeated five to ten times. Each experiment recorded the forces for $60 \mathrm{~s}$, or $120 t_{c}$. The acceleration time was $t_{\text {accel }}=0.50 t_{\mathrm{cp}}$. The case where the plate approached from above and changed direction $0.5 c_{a}$ above the test article is the particular focus of this paper. This was chosen because these vorticity fields were simpler to interpret visually than cases where the vortex impacted the test article directly, and the vortex was close enough to have a strong effect on the flow around the test article.

\section{E. Diagnostics}

PIV was used to measure velocity fields around the tunnel's midline $75 \mathrm{~cm}$ downstream of the gust generators using a LaVision PIV system. Images were captured with two Photron Fastcam APS$\mathrm{RX}$ cameras, with a resolution of $1024 \times 1024$ pixels. A $25 \mathrm{~mJ}$ DM20-527 Photonics Yttrium aluminium garnet laser was used for illumination. The laser beam was expanded into a sheet in the $x-y$ plane of the tunnel at its $z$ midpoint, using a cylindrical or Powell lens, to illuminate neutrally buoyant $10 \mu \mathrm{m}$ hollow glass beads in the water. A Powell lens was used in most experiments.

Each experiment used identical PIV parameters, apart from the first heaving gust-generation experiment, $\mathrm{HGG}-1$, which is described later. The cameras captured 2048 images in sequence at $200 \mathrm{~Hz}$. Velocity fields were computed from the raw images using LaVision DaVis 8 . This was accomplished for each set of images in three steps: mean subtraction, velocity computation, and vector merging. First, to remove the background illumination, the average intensity of the data over time was subtracted from each image. Velocity fields were then computed from each pair of mean-subtracted images with a multipass scheme. The first pass used square windows of 128 by 128 pixels, overlapping by $50 \%$. The second pass used 32-by-32 pixel circular windows with $50 \%$ overlap. These vector fields were then stitched together appropriately to show the total field of view. Spurious values of both velocity components were removed via median filters at each spatial point over ten time steps. The parameters of experiment HGG-1 were nearly identical, except that a cylindrical lens was used to spread the laser, the cameras captured 1024 images at $250 \mathrm{~Hz}$, and Davis 7 was used to compute the velocity fields. Both processings yielded a grid of velocity vectors with a vertical and lateral spacing of $0.03 c_{a}$.

An ATI Industrial Automation Nano43 force transducer was used to measure the forces and torques on the airfoil over time. The Nano43 was calibrated to a range of $\pm 36 \mathrm{~N}$ of $x-y$ force and $\pm 0.5 \mathrm{~N} \cdot \mathrm{m}$ of torque. Expressed in terms of the airfoil's force coefficients, this yielded a measurement resolution of 0.008 units, or approximately $0.8 \%$ of the maximum measured lift coefficient $C_{L}$. A Newport 481-A rotary stage was mounted between the force transducer and airfoil, allowing precise manual control of the airfoil's angle of attack.

The gantry control system recorded the positions of the gantry axes over time as well as data from the force sensor on the airfoil. It also allowed precise triggering of the PIV system, ensuring synchronized force and PIV measurements. The trajectory of the gantry was specified, and forces were measured, at time steps of $0.005 \mathrm{~s}$.

\section{F. Analysis Techniques}

1. Vortex Identification

In each set of PIV experiments, the $\Gamma_{2}$ function [41] was used to identify the shed vortices using a radius of integration of $0.6 c_{a}$. The 
circulation of the identified vortices was calculated through integration of the vorticity within the $\left|\Gamma_{2}\right|<2 / \pi$ region. The measured circulation was essentially unchanged when the radius of integration was larger than $0.4 c_{a}$. Above this threshold, there was variation in the median value of circulation of less than $\pm 10 \%$.

\section{Spatial Unwrapping of Gusts}

Downstream of the gust generators, the structures identified using the $\Gamma_{2}$ criterion were found to convect with a nearly constant velocity equal to the freestream value. This was measured by tracking the position of the peak value of $\Gamma_{2}$ over time or the vorticity-weighted centroid of the identified vortices. Both of these methods agreed consistently. The constant velocity allows the flow to be unwrapped into a larger field of view, converting a timevarying two-dimensional measurement of part of the flowfield into a single snapshot of the flow that encompasses the observed time span, as if the observer is in a comoving frame with the gust. This is performed solely for visualization purposes, as an alternative to displaying the limited field of view from the PIV system, which is only $3.5 c_{a}$ wide. More concretely, for each velocity field at time $t$, the convection-frame position of that view is computed as $x^{\prime}=x-t U$. The estimated structure is then assembled by averaging together each frame, interpolated onto its convection-frame position. With the freestream velocity subtracted, the result is an expanded view of the convecting structure in its own frame. A schematic of this process is shown in Fig. $\underline{3}$, showing only three snapshots for clarity. Note that $t<0$ is to the right, because flow moved to the right. The later figures that display images of the generated gusts were created using this method.

\section{Filtering and Force Envelopes}

The forces measured in these experiments have been decomposed into two parts: low-frequency variation due to the flow around the gust generator (below $1.12 \mathrm{~Hz}$ ) and the magnitude of higherfrequency oscillations that are primarily due to vortex shedding from the test article (between 1.12 and $10 \mathrm{~Hz}$ ). The plots later in this Paper display the averages of these quantities across the repetitions of each experiment. This decomposition was performed in MATLAB ${ }^{\circledR}$ using zero-phase digital filtering with eighth-order Butterworth filters.

Before the forces were decomposed, the effects of the tunnel's pump (operating at $12.5 \mathrm{~Hz}$ ) were removed via a low-pass filter at $10 \mathrm{~Hz}$. For comparison, the bluff-body shedding frequency for a body as thick as this airfoil at $\alpha=0 \mathrm{deg}$ is approximately $2 \mathrm{~Hz}$, which is much lower than the filter frequency.

The magnitude of the higher-frequency oscillations is referred to as the force envelope. It is important to examine the envelope, rather than the instantaneous high-frequency oscillations, because the phase of the vortex shedding process varies across experiments. The variation of the envelope over time shows when vortex shedding is reduced or at a steady state. These envelopes were calculated as the absolute value of the MATLAB®-computed Hilbert transform of the higher-frequency force oscillations.

The results are relatively insensitive to changes in the lowfrequency filter over the range of $0.95-1.7 \mathrm{~Hz}$ and to the highfrequency filter over the range of $8-10.5 \mathrm{~Hz}$.

\section{Inviscid Estimates of Vortical Gust Circulation}

Using inviscid thin airfoil theory, conservation of circulation [42], the Kutta-Joukowski theorem, and the Wagner function and assuming instantaneous changes in the direction of motion or angle of attack of the gust generators, the strength of the generated vortices may be estimated as

$$
\begin{aligned}
\Gamma_{v, \text { est,pitch }} & \approx \frac{1}{2}\left(\alpha_{2}-\alpha_{1}\right) \pi c_{a} U, \\
\Gamma_{v, \text { est,heave }} & \approx \pi c_{p} S U
\end{aligned}
$$

These equations suggest that the circulation of the generated vortices should roughly scale linearly with the freestream speed, chord length, and change in angle of attack or heaving speed of the generator. These simple estimates will be used to normalize the measured gust circulations.

\section{Gust Characterization}

\section{A. Pitching Generator Gusts}

Figure 4 shows the mean values of the unwrapped (spanwise) vorticity, normalized with the chord length and freestream velocity, that resulted from pitching the PGG to each of three final angles of attack: $\alpha_{2}=-5,-10$, and -13 deg. Because of the spatial nondimensionalization with respect to the airfoil chord and the constant convection velocity of the vortices, $x^{\prime} / c_{a}$ is equivalent to $-t / t_{c}$ for the airfoil. Thus, the region to the right of $x^{\prime}=0$ is associated with the angle of attack of the PGG before pitching, i.e., $\alpha_{1}$, and to the left with $\alpha_{2}$, after pitching. Between these, near $x^{\prime} / c_{a}=0$, is the vortex shed by the PGG. In the -5 deg case, the primary vortex is difficult to find with the naked eye. In the other tests, the vortex is clearly visible.

The streamlined profile of the static PGG resulted in a weak wake when the PGG was at $-5 \mathrm{deg}$ and a slower and more unsteady wake at -10 and $-13 \mathrm{deg}$. At $-13 \mathrm{deg}$, the wake shifted downward $6-8 t_{c}$ after the vortex passed $\left(x^{\prime} / c_{a}\right.$ of -6 to -8$)$. This was likely a result of the flow around the PGG adjusting to its new angle. The wake thus had three portions: the initial wake before pitching, the transitional wake immediately after pitching, and the final wake due to the PGG's new angle of attack. The increased wake thickness and unsteadiness after pitching suggests that the effect of the vortex on a test article may be difficult to separate from that of the PGG's changing wake.

\section{B. Heaving Generator Gusts}

A subset of the unwrapped vorticity fields from experiment HGG-1 is shown in Fig. 5 for a range of heaving speed ratios $S$ and both chord lengths. Note that the active flow disturbance had a shorter duration for these cases, and hence the recording time of these experiments was shorter than others; thus, a smaller range of $x^{\prime}$ data is available, but the same scale is used to enable comparisons between the HGG and PGG. These gusts consisted of two portions: the primary vortex at the center and the V-shaped wake region outside of it. This shape was due to the path of the HGG as it advanced and retreated across the test section, beginning and ending near the
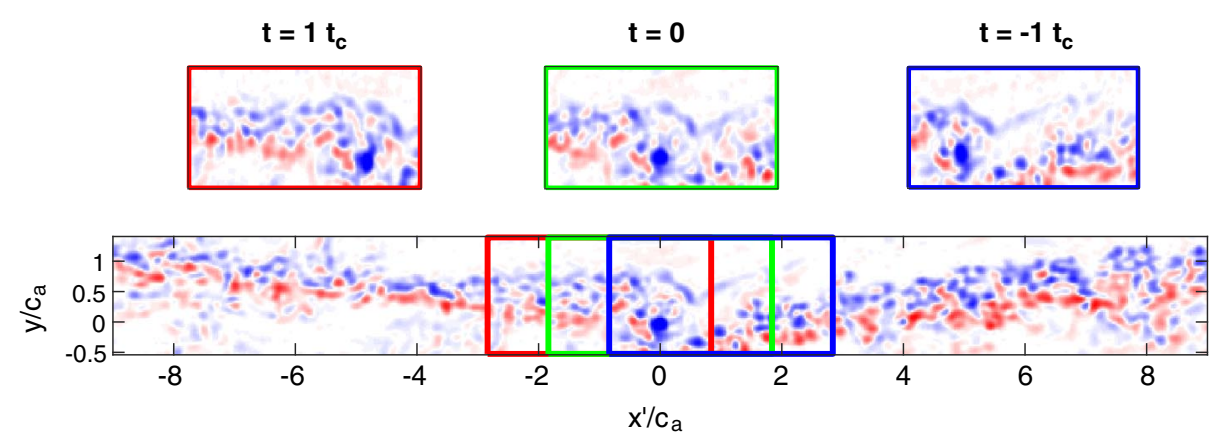

Fig. 3 Illustration of the unwrapping process, in which the flow convects from left to right. Here, $S=0.09, c_{p}=5 \mathrm{~cm}$, and $y_{\text {peak }}=0$. 

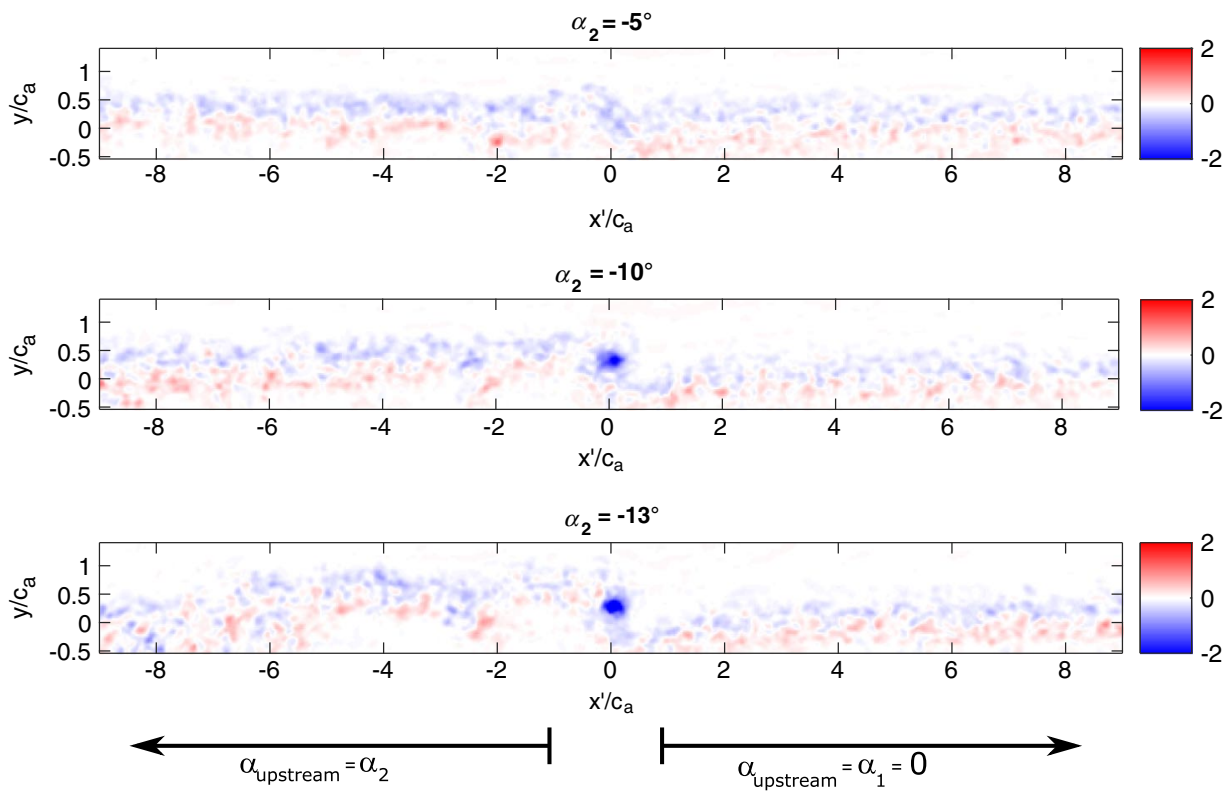

Fig. 4 Unwrapped vorticity downstream of the PGG after pitching from $\alpha_{1}=0$ deg to $\alpha_{2}=-5,-10$, or -13 deg. Vorticity has been normalized by $U / c_{a}$.
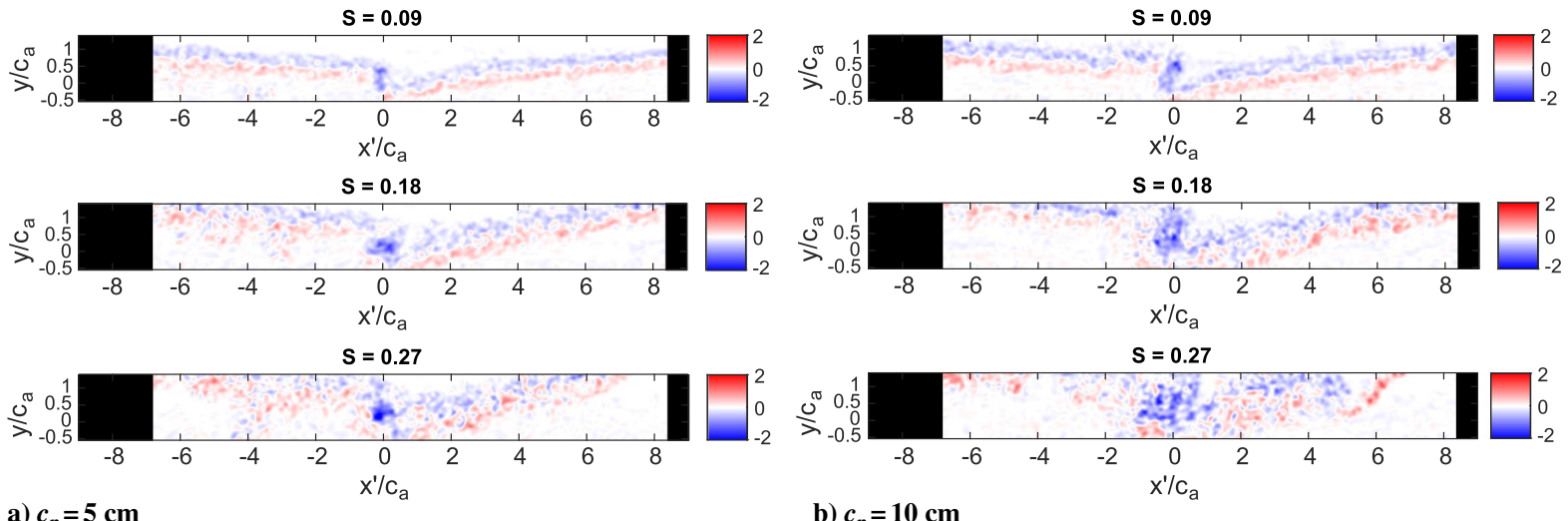

Fig. 5 Unwrapped vorticity of the gusts $75 \mathrm{~cm}$ downstream of the HGGs with 5 and $10 \mathrm{~cm}$ chords. Each row is a different $S$.

$+y$ side of the tunnel. With either of the plates, increasing $S$ led to larger and more energetic primary vortices as well as more energy in the trailing wake regions. The use of the longer plate generally resulted in more complex flow patterns in both the wake and vortex regions.

With the shorter plate at the two higher speeds, the wake appeared to temporarily broaden around $x^{\prime} / c_{a}=-4$. This vorticity was likely shed from the plate as the flow around it adjusted to its transverse velocity.

Of note is the limited region of freestream perturbations from the HGG when it was far from the midline, i.e., when docked at the side wall, and the increased $y$ offset of the HGG wake relative to the primary vortex in comparison with the PGG vorticity fields of Fig. 4.

The motion parameters $S$ and $T$ had large effects on the flow around the plate. The heaving speed $S$ determined the effective angle of attack, $\alpha_{\text {eff }}=\arctan (S)$, of the HGG in motion. In the experiments in which $\alpha_{\text {eff }} \approx 5 \operatorname{deg}(S=0.09)$, the plate's wake was consistent with attached flow, as would be expected with a static flat plate at that angle of attack. Analogously, the wake was consistent with separated flow when the plates moved at $\alpha_{\text {eff }} \gtrsim 10 \operatorname{deg}(S \gtrsim 0.18)$. These experiments with high $\alpha_{\text {eff }}$ also required more time for the flow around the plate to settle into regular vortex shedding, which is consistent with developing flows over airfoils that rapidly pitched to high angles of attack. When the dimensionless heaving time $T$ was large, the flow around the plate had enough time to approach its final behavior of regular vortex shedding before the direction change. This was seen in experiments with low $S$, which thus took more time to reach the center of the tunnel. When $T$ was too small, the flow did not have enough time to develop and so was still evolving when the plate changed directions. This resulted in weaker and less well-organized primary vortices.

\section{Comparison of Gust Generators}

Schematics of the evolving flows around each generator are shown in Fig. 6, including the difference in the position of their wakes. For the PGG, the top frame of Fig. 6 a shows the airfoil before pitching, with regular vortex shedding behind it. In the center, the airfoil is in the middle of pitching, with a vortex rolling up near the trailing edge. In the bottom and final frame, the vortex is convecting downstream, as the flow around the airfoil establishes itself. For the HGG, the top frame of Fig. $6 \mathrm{~b}$ shows the plate moving downward with a wake of regularly shed vortices. In the second, the plate has just reversed direction and is forming a vortex on its lower surface. In the third frame, the plate continues moving upward as the primary vortex convects downstream.

Figure 7 shows metrics of the different gusts from both the PGG and HGG (experiment HGG-2). For comparison with the unwrapped gusts, the horizontal axis is the spatial coordinate $x^{\prime} / c_{a}$. In the presented data, both the $y_{\text {peak }}$ position of the HGG and $y_{\text {upstream }}$ for the PGG are at $y=0$. The gusts have been averaged in the $y$ direction and across multiple repetitions of each experiment, yielding comparisons of the dimensionless streamwise velocity $u$, transverse velocity $v$, vorticity $\omega_{z}$, kinetic energy $\left(u^{2}+v^{2}\right) / U^{2}$, and enstrophy $\omega_{z}^{2}$. Velocities are described in the frame of the convecting gust. As 


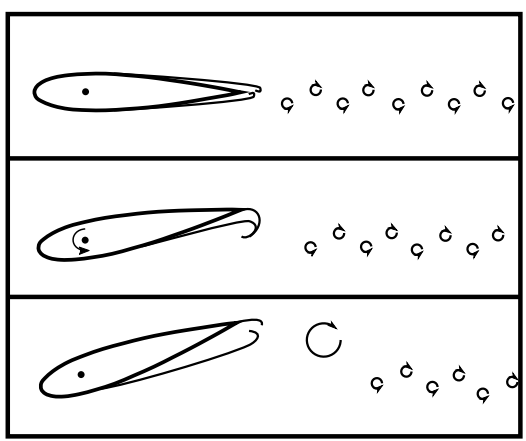

a) Action of PGG

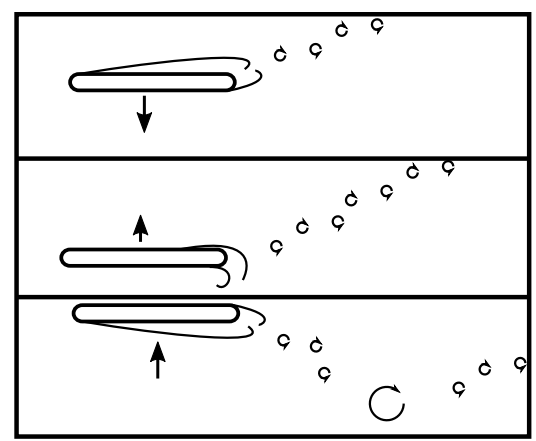

b) Action of HGG

Fig. 6 Sequential sketches of the generation of a vortical gust by the PGG and HGG.

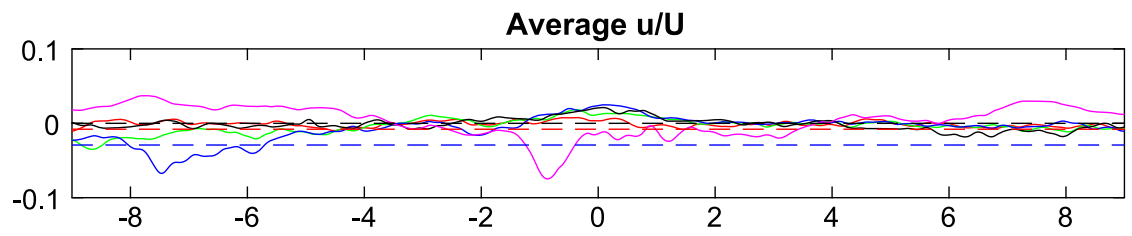

Average v/U

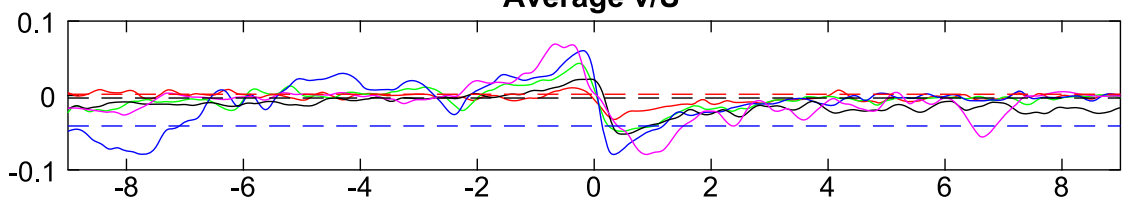

Average $\omega_{\mathrm{z}} /\left(\mathrm{U} / \mathrm{c}_{\mathrm{a}}\right)$

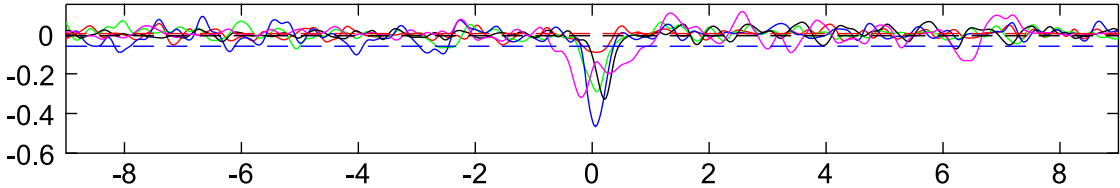

Average KE / U ${ }^{2}$

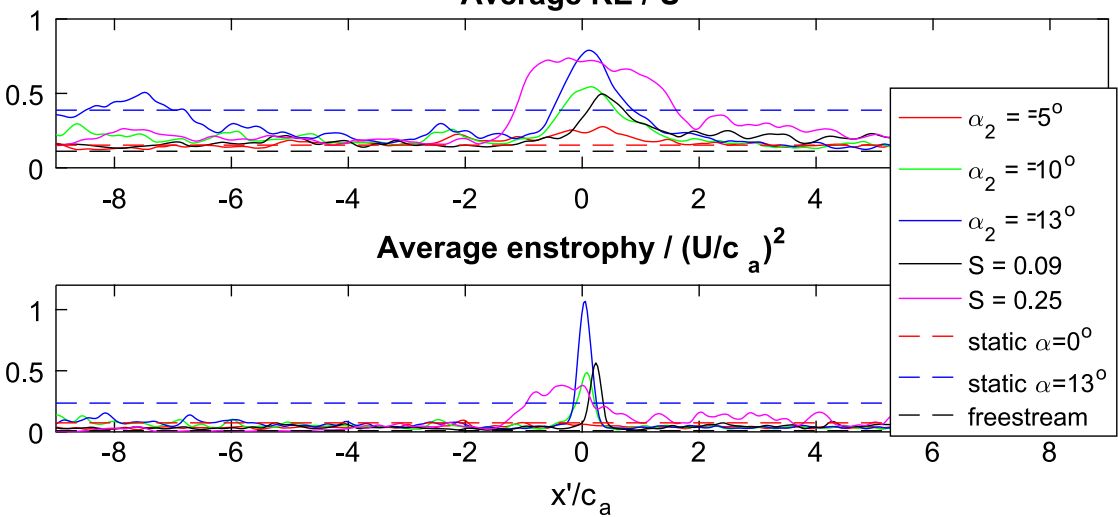

Fig. 7 Spatial profiles of the $y$-averaged gusts created with the $5 \mathrm{~cm}$ HGG and $10 \mathrm{~cm}$ PGG mechanisms.

baselines, the same quantities have been plotted for the static PGG as well as the unperturbed freestream. For comparison, the experimental circulations of the primary vortices in each of these experiments are shown in Table $\underline{1}$, sorted by magnitude.

Table 1 Experimental circulations of the vortices compared in Fig. 7 (the PGG's airfoil has $c_{a}=10 \mathrm{~cm}$, and the HGG's plate has $c_{p}=5 \mathrm{~cm}$ )

\begin{tabular}{lcc}
\hline \hline Apparatus & Plot label & Median $\Gamma_{v} \cdot 10^{-3}, \mathrm{~m}^{2} / \mathrm{s}$ \\
\hline PGG & $\alpha_{2}=-5 \mathrm{deg}$ & -1.9 \\
HGG & $S=0.09$ & -4.1 \\
PGG & $\alpha_{2}=-10 \mathrm{deg}$ & -5.1 \\
HGG & $S=0.25$ & -7.3 \\
PGG & $\alpha_{2}=-13 \mathrm{deg}$ & -7.4 \\
\hline \hline
\end{tabular}

The $v$ velocity profile shows the expected increase then decrease in speed associated with a vortex. Comparison with the vorticity profile shows that there is indeed a vortical region there. The kinetic energy and enstrophy plots show the expected peaks at the vortex.

The vortex shed by the PGG with $\alpha_{2}=-5$ deg is barely distinguishable from the background unsteadiness of the flow. The other vortices shed by the PGG are spatially compact, compared to those of the HGG. This is particularly true with comparison to the $S=0.25$ vortex, which is spatially broad in both enstrophy and kinetic energy. The plots shown have not been scaled with respect to the chord lengths of the two devices; doing so would double the relative width of the gusts from the HGG.

The wakes of the devices can be most readily compared in the kinetic energy plot. Seven convective time units after pitching, or $x^{\prime} / c_{a} \lesssim-7$, the unsteadiness of the PGG's wake at $-13 \mathrm{deg}$ 


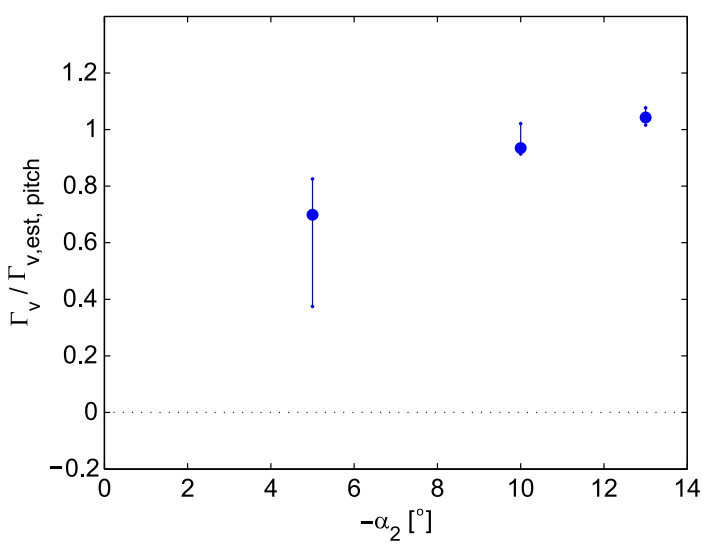

a) Normalized circulation from PGG

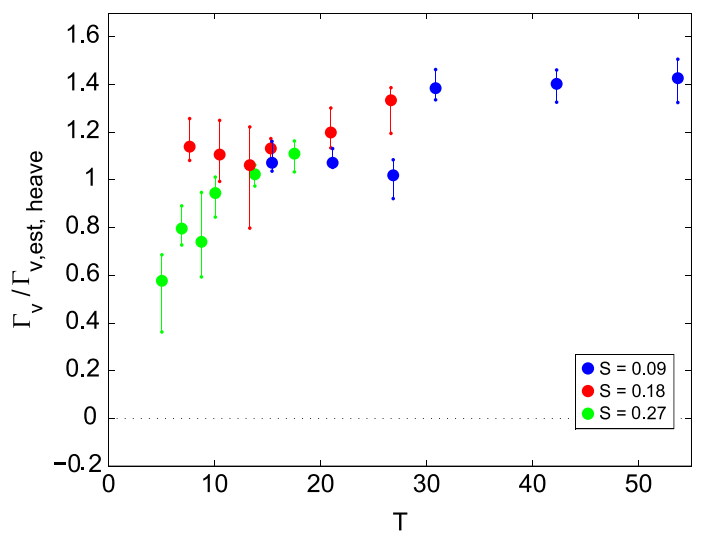

b) Normalized circulation from 5 and $10 \mathrm{~cm}$ HGGs

Fig. 8 Median, 25th, and 75th percentile measures of the normalized circulation of the vortices shed by the gust generators.

matched that of the static airfoil at the same angle. In contrast, the wake of the HGG left the field of view by this point and so no longer disturbed the measured flow.

The circulation of the primary vortex, normalized by the thin airfoil theory estimates [Eq. (6)], is plotted in Fig. 8 against the final angle of attack for the $\mathrm{PGG}$ and against the dimensionless heaving time for the HGG (measured in experiment HGG-1). The large symbols are the median values of the circulation, and the whiskers show the 25 th and 75 th percentiles. A value of 1 would denote exact agreement of the experimental data with inviscid theory, but substantial variation is observed. With the PGG, the $\Gamma_{2}$ function was not always able to satisfactorily identify the weakest vortices due to the strong influence of the wake, so the variance in circulation is large for $\alpha_{2}=-5 \mathrm{deg}$. With regard to the HGG, the experiments with small $S$ (or large $T$ ) tended to generate relatively stronger vortices. Shorter heaving times generally reduced the normalized strength of the vortices, but this was confounded by the heaving speed and plate length.

To summarize, both approaches to gust generation were able to create repeatable and clearly identifiable vortical gusts that convected downstream using only a single degree of actuation. Both the PGG and the $5 \mathrm{~cm}$ HGG at $S=0.09$ created spatially compact vortical gusts, as compared to those of the $10 \mathrm{~cm} \mathrm{HGG}$, which were larger and had less distinct cores. Less power was needed to pitch the PGG, compared to the power needed to rapidly reverse the direction of the HGG. The PGG had the downside of remaining in place and continually disturbing the flow with its wake, whereas the HGG moved its wake such that it no longer perturbed the test article.

\section{Gust-Airfoil Interaction}

\section{A. Interaction with PGG Gusts}

An example of the interaction between the airfoil and a gust from the PGG is shown in Fig. 9. At the top are four snapshots of the vorticity field for increasing time, in which the primary vortex and wake from the gust generator are passing the airfoil. The $\Gamma_{2}$ criterion has been used to outline the vortical regions in black. The second row is a plot of the experimental lift coefficients over time, with the temporal position of the vorticity snapshots denoted by green circles at $\tau_{a}=[-1,0,1,6]$. Please note that time here increases to the right, in contrast to the "unwrapped" figures. The red lines show the lowpass-filtered $C_{L}$ from each repetition of the experiment. The black line is the average of those forces. The dashed blue lines show the average $C_{L}$ envelope around the average $C_{L}$. The final plot shows the angle of attack of the PGG as a function of time, as well as this angle translated in time by the travel time of the gust, indicating the expected arrival time of the vortex at the airfoil, $x=0$.

In the vorticity snapshots of Fig. 9, the primary vortex is visible as the strongly negative region, which is about one chord length above the airfoil and outlined in black. Across the first three images, it traveled from just upstream of the airfoil to behind its trailing edge. Also visible
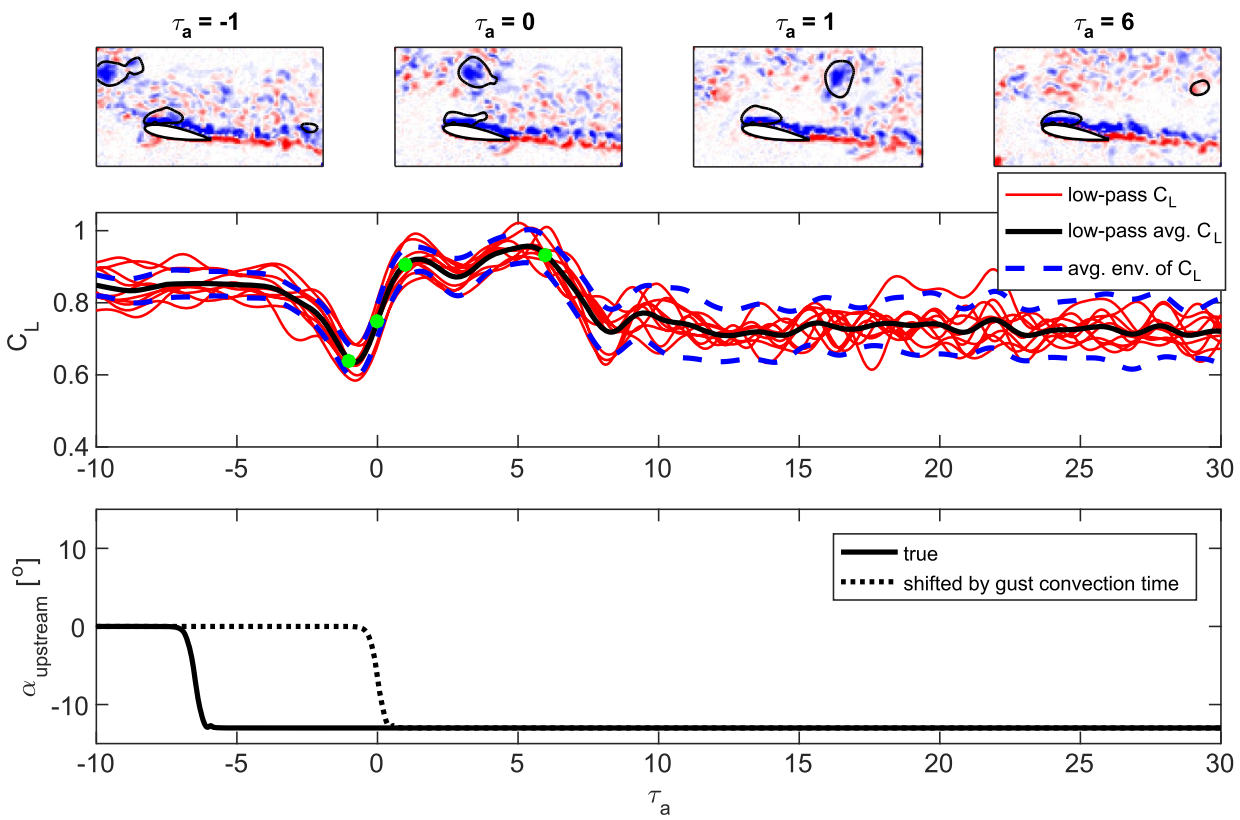

Fig. 9 Gusts from the PGG interacting with the downstream airfoil: $y_{\text {upstream }}=0.5 c_{a}, \alpha_{2}=-13$ deg, and $\alpha=10$ deg (avg., average; env., envelope). 
are complex, small-scale vortical structures that are associated with the wake of the PGG. Additionally, 5-10 $t_{c}$ after the vortex reached the airfoil, the wake appears to shift downward. This is visible on the left edge of the fourth image. The variations in the low-pass filtered forces appear to coincide with two main factors: the incoming vortex and the change in the oncoming wake before and after the vortex.

With regard to the vortex-associated forces, the low-pass-filtered $C_{L}$ curves followed similar trends in each experiment. In response to a vortex with negative circulation, the lift initially dropped as the vortex approached the leading edge, then rebounded above the steady-state value as the vortex passed over the airfoil, and slowly approached its steady-state value as the vortex traveled farther downstream. The opposite occurred in response to a vortex with positive circulation.

The difference in the PGG's pre- and postpitching wake significantly modified the forces on the test article. Both the average value and magnitude of oscillation of those forces changed permanently. $C_{L}$ dropped by approximately 0.1 , and the magnitude of oscillation grew by a factor of nearly 2.5 . This was far from the ideal of a single transient interaction with a vortex. In this experiment, the wake of the PGG at $\alpha=0 \mathrm{deg}$, or $\tau_{a}<0$, impacted the test article asymmetrically, resulting in a higher value of $C_{L}$ and a reduction in von Kármán vortex shedding. This reduction is visible as a narrower force envelope. After pitching, the PGG's wake was farther from the airfoil, resulting in lower lift and more vortex shedding, which is visible as the wider force envelope after the gust interaction. The increase in the magnitude of the force envelope was slightly counterintuitive, as the airfoil experienced more freestream perturbations before the vortex interaction, but these perturbations reduced the overall variation in force by damping regular vortex shedding. The effects of the PGG's wake were not identical with every variation in the position and angle of the PGG and test article, but had a weak dependence on the final angle of the generator and its relative position.

Overall, the PGG was not a perfect gust generator. Although it was able to generate compact vortical gusts, its persistent presence upstream of the test article made it difficult to separate the effects of its wake from those of the vortex. Beyond the initial lift peak, it was difficult to ascribe further effects solely to the passing vortex. This suggests that the PGG may be inappropriate to use for examining solitary vortex-wing interactions when it is nearly directly upstream of the test article, as its unwanted wake has strong effects.

\section{B. Interaction with HGG Gusts}

In contrast to the prepitching PGG, the $5 \mathrm{~cm}$ HGG held at its initial position had no noticeable effect on the test article's forces. The results of a representative dynamic experiment with the HGG (from experiment HGG-3) are shown in Fig. 10, in which the HGG approached the airfoil from above. This is analogous to Fig. 9, but with the HGG's position over time in the bottommost plot. In these experiments, compared to those in Fig. 9 , the initial $C_{L}$ was lower and had a higher level of fluctuations. The lower level of freestream perturbations in these experiments resulted in the lower $C_{L}$, and the fluctuations were due to von Kármán vortex shedding from the test article.

The first three PIV snapshots in Fig. 10 show the outlined vortical gust above the airfoil, traveling from left to right. The fourth snapshot shows the wake during its withdrawal near the upper edge of the field of view as well as the relatively calm flow directly upstream of the airfoil. Because the wake of the HGG followed its $y$ position, it was only visible in the PIV field of view for a limited amount of time. After the gust passed, the flow and forces returned to their initial state.

As with the gust from the PGG, the forces due to the gust from the HGG were fairly repeatable during the closest approach of the vortex. In response to an oncoming vortex with negative circulation, the lift coefficient dipped, rose, and returned to the steady-state value. The opposite occurred with vortices of positive circulation.

When the flow around the airfoil was sufficiently perturbed, the amplitude of the envelopes returned to their original state after approximately $15 t_{c}$, as the vortex shedding resumed. Examples of this resumption of shedding are shown in Fig. 11 for two values of $S$. In this figure, the plate approached from below the test article and changed directions $0.5 c_{a}$ below it.

Further experiments with the HGG approaching from below are presented in Fig. 12. This shows the effects of the HGG's wake on the vortex shedding process after having traveled different distances, with $\alpha=5 \mathrm{deg}$ and $S=0.09$. The force envelopes are presented because the changes in vortex shedding are not evident in the low-frequency $C_{L}$ measurements. For $y_{\text {peak }}<0$, the HGG did not pass directly ahead of the airfoil, and the force envelopes were not substantially perturbed. At $y_{\text {peak }}=0$, the vortex was released directly upstream of the airfoil, and the strong effect of the vortex could be seen as the disturbance near $\tau_{a}=0$. For $y_{\text {peak }}=1 c_{a}$, the HGG passed the airfoil twice. The wake first impacted the airfoil at $\tau_{a} \approx-10$, where the reduction of the $C_{L}$ envelope indicated a substantial disruption of vortex shedding. The wake again impacted the airfoil at $\tau_{a} \approx 10$, and the forces slowly returned to their undisturbed behavior.

The airfoil's angle of attack had a significant effect on the timevarying forces, as shown in Fig. 13. In this figure, the $C_{L}$ traces were due to gusts from the HGG moving at $S=0.09$ from the $+y$ side of the tunnel to $y_{\text {peak }}=0.5 c_{a}$, with the test article at different angles of attack. This shows the increasing recovery time with an increasing
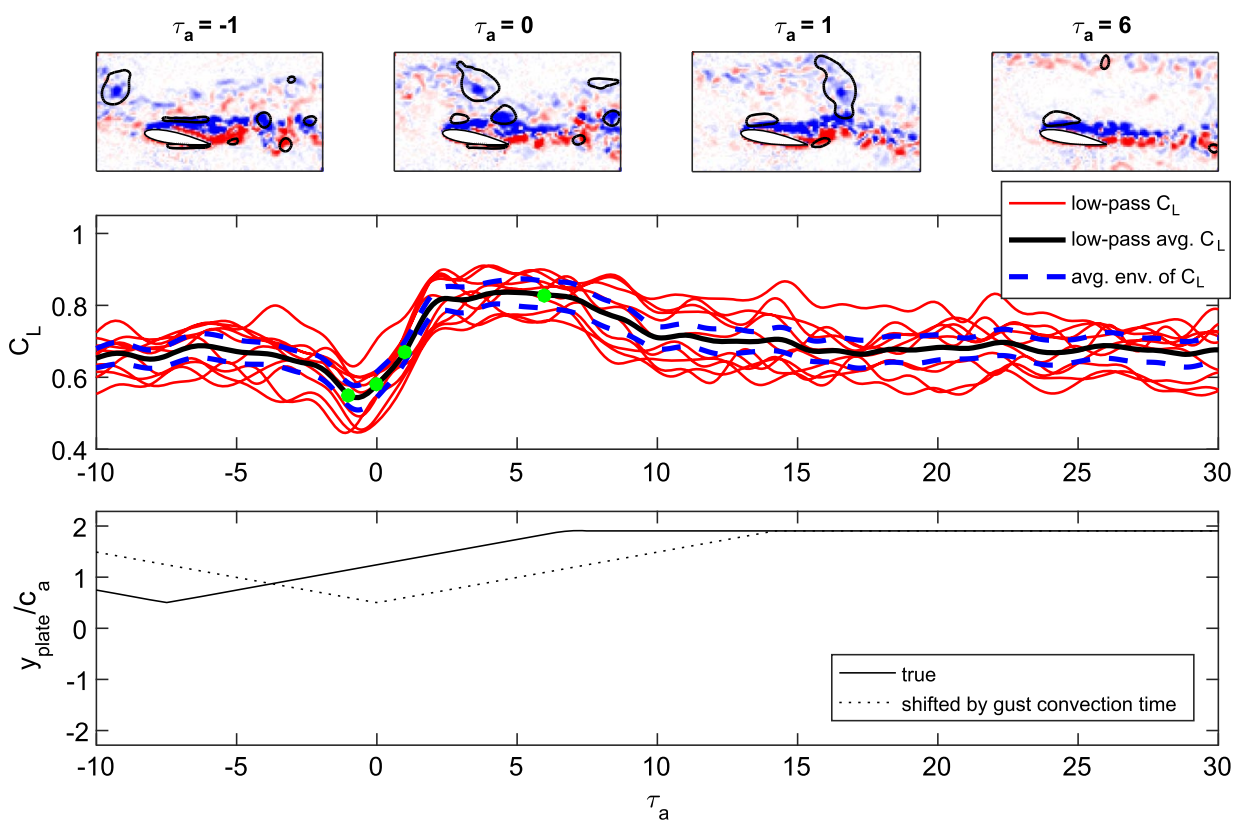

Fig. 10 Gusts from the HGG interacting with the downstream airfoil: $y_{\text {peak }}=0.5 c_{a}, \alpha=10 \mathrm{deg}, S=0.09$, and $T=28$. 


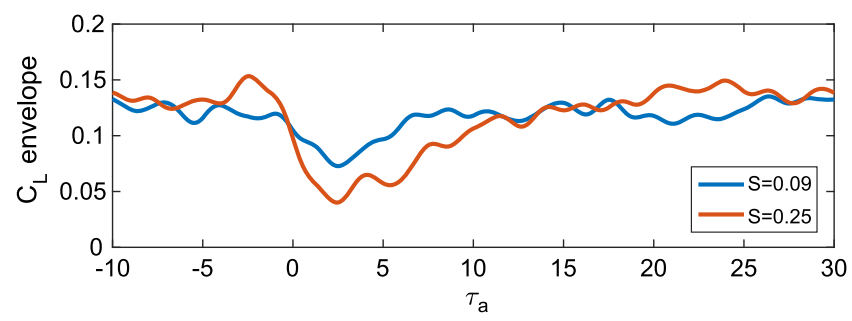

Fig. 11 Amplitude of the force envelopes corresponding to the resumption of vortex shedding after interaction with gusts from the $5 \mathrm{~cm}$ HGG, with $y_{\text {peak }}=-0.5 c_{a}$ and $\alpha=5 \mathrm{deg}$.

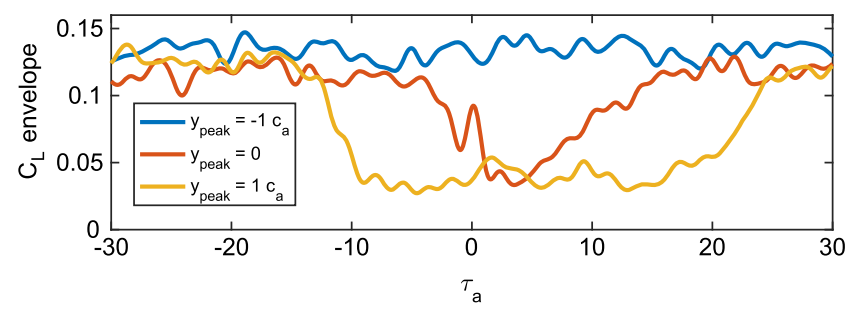

Fig. $12 C_{L}$ envelope due to the $5 \mathrm{~cm}$ HGG moving to different peak positions from below the airfoil, with $S=0.09$ and $\alpha=5 \mathrm{deg}$.

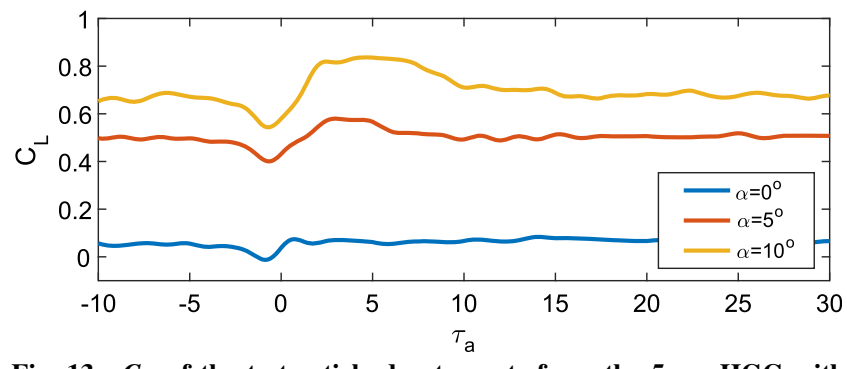

Fig. $13 C_{L}$ of the test article due to gusts from the 5 cm HGG with $S=0.09$ and $y_{\text {peak }}=0.5 c_{a}$.

angle of attack. Early in the interaction, the changes in $C_{L}$ were similar across $\alpha$, but they diverged after the first dip. At higher $\alpha$, the gusts yielded larger and more lasting deviations in the forces. The recovery time was approximately $5-10 t_{c}$ for the lower angles and $10-20 t_{c}$ for $\alpha=10 \mathrm{deg}$.

The multiple time scales for the return of the flow to its original state suggest multiple causes. The rapid recovery of the low-passfiltered forces for $\alpha=0 \mathrm{deg}$ is consistent with the physics underlying the Wagner function, which suggests that the recovery is due to the release of vorticity into the wake. When the gust strongly interacted with the airfoil at $\alpha=10 \mathrm{deg}$, the flow reattached and required additional time to redevelop its separated flow. Similarly, the $\alpha=5 \mathrm{deg}$ airfoil needed an intermediate amount of time to recover from strong perturbations.

Overall, the heaving gust generator is imperfect. Although its wake only interacted with the test article for a finite time, and only when the HGG passed directly in front of the test article, such interactions are nonideal. This suggests that it is inappropriate to use for vortex generation when it would pass in front of the test article. Unfortunately, this constrains the polarity of the generated vortices to gusts with negative circulation above the airfoil and positive circulation below it.

\section{Conclusions}

The research described in this Paper aimed to better understand the generation of vortical gusts and their effects on an airfoil. This consisted of two topics: experimental generation of the gusts by pitching an airfoil or heaving a flat plate and the interaction between these gusts and a fixed downstream airfoil.

Both experimental methods were able to generate repeatable vortical gusts through actuation of a single degree of freedom but also created potentially intrusive wakes with differing characteristics. The pitching gust generator, since it remained in place upstream of the airfoil, continually released an inconvenient wake behind it. In contrast, the heaving gust generator moved to the edge of the tunnel, limiting the time that its wake influenced the flow around the test article. However, the vortical gusts from the heaving gust generator (HGG) were less spatially compact than those of the pitching gust generator (PGG).

Both types of generated vortices caused similar effects on a downstream airfoil: an initial extreme in lift as the vortex passed the airfoil's leading edge, then an opposite overshoot, and a slow approach to the final force states. The wakes of the devices also induced substantial effects, which limited the usefulness of both. In some cases, the PGG's wake changed the flow around the test article to such an extent that the average forces were significantly different before and after pitching. If the PGG was directly upstream of the test article, it also reduced the coherent vortex shedding from the test article when it was at moderate angles of attack. This suggests that it should only be used when it is far (in the $y$ direction) from the test article. The HGG was conceived to reduce the effect of the generator's wake on the test article and was shown to not affect the test article when it was kept by the side of the tunnel. When the HGG passed in front of the test article, its wake impacted the test article twice with significant effects on the flow and forces. Avoiding this interaction limits the polarity of the HGG's shed vortices: those with positive circulation below the airfoil and negative above it.

The angle of attack of the airfoil had a significant impact on the time needed for the low-frequency forces to transition from their perturbed levels to their final states. When the airfoil was at $\alpha=0 \mathrm{deg}$, the forces approached their final state quickly, on a time scale of $5-10 t_{c}$. When the airfoil was almost statically stalled at $\alpha=10 \mathrm{deg}$, large perturbations from the gust could cause the flow to temporarily reattach, resulting in recovery time scales of up to $20 t_{c}$. This is consistent with the time scales reported for high-Reynoldsnumber vortex-wing interactions [38], in the control of separated flows [43], and the forces on rapidly accelerated plates [44].

Examination of the higher-frequency force oscillations provided insight into the vortex shedding process. Use of the HGG yielded one result unavailable with the PGG: the timescale of resumption of vortex shedding from the airfoil at $\alpha=5 \mathrm{deg}$ after a strong perturbation was $15 t_{c}$. By moving the generator's wake away from the test article, the vortex shedding process resumed its unperturbed behavior. In contrast, the wake of the PGG continued to interact with the test article and overwhelmed the re-development of the vortex shedding behavior.

Neither vortex-generation technique is perfect for the generation of isolated gusts, but the two methods could be combined in a generator that both pitches and heaves. Such a device would be more complex but could potentially both move its wake away from the test article and create compact gusts.

\section{Acknowledgments}

The authors would like to thank the reviewers for their help in significantly improving this manuscript. This research was funded by the Gordon and Betty Moore Foundation through grant GBMF\#2645 to the California Institute of Technology.

\section{References}

[1] Galway, D., Etele, J., and Fusina, G., "Development and Implementation of an Urban Wind Field Database for Aircraft Flight Simulation," Journal of Wind Engineering and Industrial Aerodynamics, Vol. 103, April 2012, pp. 73-85. doi:10.1016/j.jweia.2012.02.010

[2] Khouli, F., Afagh, F. F., and Langlois, R. G., "Design, Simulation, and Experimental Results for Flexible Rotors in a Ship Airwake," Journal of Aircraft, Vol. 53, No. 1, 2016, pp. 262-275. doi:10.2514/1.C033322

[3] Spalart, P., "Airplane Trailing Vortices," Annual Review of Fluid Mechanics, Vol. 30, No. 1, 1998, pp. 107-138. doi:10.1146/annurev.fluid.30.1.107

[4] Hesse, H., and Palacios, R., "Dynamic Load Alleviation in Wake Vortex Encounters," Journal of Guidance, Control, and Dynamics, Vol. 39, 
No. 4, 2016, pp. 801-813.

doi:10.2514/1.G000715

[5] Rockwell, D., "Vortex-Body Interactions," Annual Review of Fluid Mechanics, Vol. 30, No. 1, 1998, pp. 199-229. doi:10.1146/annurev.fluid.30.1.199

[6] Eldredge, J. D., and Jones, A. R., "Leading-Edge Vortices: Mechanics and Modeling," Annual Review of Fluid Mechanics, Vol. 51, No. 1, 2019, pp. 75-104. doi:10.1146/annurev-fluid-010518-040334

[7] Donely, P., "Summary of Information Relating to Gust Loads on Airplanes," NACA TR 997, 1949.

[8] Etele, J., "Overview of Wind Gust Modelling with Application to Autonomous Low-Level UAV Control," Defence Research and Development Canada-Ottawa, CR 2006-221, 2006.

[9] Dryden, H., "A Review of the Statistical Theory of Turbulence," Quarterly of Applied Mathematics, Vol. 1, No. 1, 1943, pp. 7-42. doi:10.1090/qam/1943-01-01

[10] Von Kármán, T., "Progress in the Statistical Theory of Turbulence," Proceedings of the National Academy of Sciences, Vol. 34, No. 11, 1948, pp. 530-539.

[11] "Dynamic Gust Loads," ANM-115, Transport Airplane Directorate, Federal Aviation Administration, Advisory Circular TR 25.341-1, 2014

[12] Knigge, C., and Raasch, S., "Improvement and Development of oneand Two-Dimensional Discrete Gust Models Using a Large-Eddy Simulation Model," Journal of Wind Engineering and Industrial Aerodynamics, Vol. 153, June 2016, pp. 46-59. doi:10.1016/j.jweia.2016.03.004

[13] Fung, Y., An Introduction to the Theory of Aeroelasticity, Courier Corp., Minneola, NY, 2002, pp. 381-417.

[14] Wagner, H., "Über die Entstehung des dynamischen Auftriebes von Tragflügeln," Zeitschrift für Angewandte Mathematik und Mechanik, Vol. 5, No. 1, 1925, pp. 17-35. doi:10.1002/(ISSN)1521-4001

[15] Theodorsen, T., "General Theory of Aerodynamic Instability and the Mechanism of Flutter," NACA Annual Report, Vol. 20, 1935.

[16] Küssner, H., "Zusammenfassender Bericht über den instationären Auftrieb von Flügeln," Lufifahrtforschung, Vol. 13, No. 12, 1936, pp. 410-424.

[17] von Kármán, T., and Sears, W., "Airfoil Theory for Non-Uniform Motion," Journal of the Aeronautical Sciences, Vol. 5, No. 10, 1938, pp. 379-390. doi: $10.2514 / 8.674$

[18] Tchieu, A., and Leonard, A., "A Discrete-Vortex Model for the Arbitrary Motion of a Thin Airfoil with Fluidic Control," Journal of Fluids and Structures, Vol. 27, No. 5, 2011, pp. 680-693. doi:10.1016/j.jfluidstructs.2011.02.008

[19] Donely, P., "An Experimental Investigation of the Normal Acceleration of an Airplane Model in a Gust," NACA TR 706, 1939.

[20] Perrotta, G., and Jones, A. R., "Unsteady Forcing on a Flat-Plate Wing in Large Transverse Gusts," Experiments in Fluids, Vol. 58, No. 8, 2017, Paper 101. doi:10.1007/s00348-017-2385-z

[21] Biler, H., Badrya, C., and Jones, A. R., "Experimental and Computational Investigation of Transverse Gust Encounters," 2018 AIAA Aerospace Sciences Meeting, AIAA Paper 2018-0571, 2018. doi:10.2514/6.2018-0571

[22] Stevens, P. R. R. J., et al., "Experiments and Computations on the Lift of Accelerating Flat Plates at Incidence," AIAA Journal, Vol. 55, No. 10, 2017, pp. 3255-3265. doi:10.2514/1.J055323

[23] Buell, D., "An Experimental Investigation of the Velocity Fluctuations Behind Oscillating Vanes," NASA TN D-5543, 1969.

[24] Poussot-Vassal, C., Demourant, F., Lepage, A., and Bihan, D. L., "Gust Load Alleviation: Identification, Control, and Wind Tunnel Testing of a 2-D Aeroelastic Airfoil," IEEE Transactions on Control Systems Technology, Vol. 25, No. 5, 2017, pp. 1736-1749. doi:10.1109/TCST.2016.2630505

[25] Kerstens, W., Pfeiffer, J., Williams, D., King, R., and Colonius, T., "Closed-Loop Control of Lift for Longitudinal Gust Suppression at Low Reynolds Numbers," AIAA Journal, Vol. 49, No. 8, 2011, pp. 1721-1728. doi:10.2514/1.J050954

[26] Harding, S., Payne, G., and Bryden, I., "Generating Controllable Velocity Fluctuations Using Twin Oscillating Hydrofoils: Experimental Validation," Journal of Fluid Mechanics, Vol. 750, July 2014, pp. 113-123. doi:10.1017/jfm.2014.257

[27] Brion, V., Lepage, A., Amosse, Y., Soulevant, D., Senecat, P., Abart, J., and Paillart, P., "Generation of Vertical Gusts in a Transonic Wind Tunnel," Experiments in Fluids, Vol. 56, No. 7, 2015, p. 145. doi:10.1007/s00348-015-2016-5
[28] Golubev, V., Hollenshade, T., Nguyen, L., and Visbal, M., "Parametric Viscous Analysis of Gust Interaction with SD7003 Airfoil," 48th AIAA Aerospace Sciences Meeting Including the New Horizons Forum and Aerospace Exposition, AIAA Paper 2010-928, 2010. doi:10.2514/6.2010-928

[29] Baik, Y., and Bernal, L., "Experimental Study of Pitching and Plunging Airfoils at Low Reynolds Numbers," Experiments in Fluids, Vol. 53, No. 6, 2012, pp. 1979-1992. doi:10.1007/s00348-012-1401-6

[30] Baik, Y., Bernal, L., Granlund, K., and Ol, M., "Unsteady Force Generation and Vortex Dynamics of Pitching and Plunging Aerofoils," Journal of Fluid Mechanics, Vol. 709, Oct. 2012, pp. 37-68. doi:10.1017/jfm.2012.318

[31] Buchholz, J. H. J., Green, M. A., and Smits, A. J., "Scaling the Circulation Shed by a Pitching Panel," Journal of Fluid Mechanics, Vol. 688, Dec. 2011, pp. 591-601. doi: $10.1017 / \mathrm{jfm} .2011 .408$

[32] Golubev, V., Hollenshade, T., Nguyen, L., and Visbal, M., "HighAccuracy Low-Re Simulations of Airfoil-Gust and Airfoil-Vortex Interactions," 40th AIAA Fluid Dynamics Conference and Exhibit, AIAA Paper 2010-4868, 2010. doi: $10.2514 / 6.2010-4868$

[33] Golubev, V., Nguyen, L., and Visbal, M., "High-Fidelity Simulations of Transitional Airfoil Interacting with Upstream Vortical Structure," 49th AIAA Aerospace Sciences Meeting Including the New Horizons Forum and Aerospace Exposition, AIAA Paper 2011-394, 2011. doi:10.2514/6.2011-394

[34] Gordnier, R., and Visbal, M., "Impact of a Vortical Gust on the Aerodynamics of a Finite Aspect-Ratio wing," 45th AIAA Fluid Dynamics Conference, AIAA Paper 2015-3072, 2015. doi:10.2514/6.2015-3072

[35] Barnes, C. J., and Visbal, M. R., "Effects of Vertical Position and Orientation on a Vortical-Gust/Airfoil Interaction at a Transitional Reynolds Number," 47th AIAA Fluid Dynamics Conference, AIAA Paper 2017-3320, 2017. doi: $10.2514 / 6.2017-3320$

[36] Nguyen, L., Golubev, V. V., and Visbal, M. R., "Numerical Study of Transitional SD7003 Airfoil Interacting with Canonical Upstream Flow Disturbances," AIAA Journal, Vol. 56, No. 1, 2018, pp. 158-181. doi:10.2514/1.J055900

[37] Barnes, C. J., and Visbal, M. R., "Gust Response of Rigid and Elastically Mounted Airfoils at a Transitional Reynolds Number," Aerospace Science and Technology, Vol. 74, March 2018, pp. 112-119. doi:10.1016/j.ast.2017.12.025

[38] Barnes, C. J., and Visbal, M. R., "Further Investigations of Vortical-Gust/Airfoil Interactions at a Transitional Reynolds Number," 2018 AIAA Aerospace Sciences Meeting, AIAA Paper 2018-0823, 2018. doi: $10.2514 / 6.2018-0823$

[39] Hufstedler, E., "Experimental Generation and Modeling of Vortical Gusts and their Interactions with an Airfoil," Ph.D. Thesis, California Inst. of Technology, Pasadena, CA, 2017. doi:10.7907/Z9Q52MN5

[40] Eldredge, J., Wang, C., and Ol, M. V., "A Computational Study of a Canonical Pitch-Up, Pitch-Down Wing Maneuver," 39th AIAA Fluid Dynamics Conference, AIAA Paper 2009-3687, 2009. doi:10.2514/6.2009-3687

[41] Graftieaux, L., Michard, M., and Grosjean, N., "Combining PIV, POD and Vortex Identification Algorithms for the Study of Unsteady Turbulent Swirling Flows," Measurement Science and Technology, Vol. 12, No. 9, 2001, pp. 1422-1429. doi:10.1088/0957-0233/12/9/307

[42] Thomson, W., "On Vortex Motion," Transactions of the Royal Society of Edinburgh, Vol. 25, No. 1, 1868, pp. 217-260. doi:10.1017/S0080456800028179

[43] Colonius, T., and Williams, D., "Control of Vortex Shedding on Twoand Three-Dimensional Aerofoils," Philosophical Transactions of the Royal Society A: Mathematical and Physical, Vol. 369, No. 1940, 2011, pp. $1525-1539$. doi:10.1098/rsta.2010.0355

[44] Mancini, P., Manar, F., Granlund, K., Ol, M. V., and Jones, A. R., "Unsteady Aerodynamic Characteristics of a Translating Rigid Wing at Low Reynolds Number," Physics of Fluids, Vol. 27, No. 12, 2015, Paper 123102. doi:10.1063/1.4936396 